\title{
Localization of overlap modes and topological charge, vortices and monopoles in $S U(3)$ LGT
}

\section{Ernst-Michael Ilgenfritz ${ }^{* a}$, Karl Koller ${ }^{b}$, Yoshiaki Koma ${ }^{c}$, Gerrit Schierholz $^{d, e}$, Thomas Streuer $^{f}$, Volker Weinberg ${ }^{d, g}$ and Markus Quandt ${ }^{h}$}

${ }^{a}$ Institut für Physik, Humboldt Universität zu Berlin, 12489 Berlin, Germany

${ }^{b}$ Sektion Physik, Universität München, 80333 München, Germany

${ }^{c}$ Numazu National College of Technology, Numazu 410-8501, Japan

${ }^{d}$ John von Neumann-Institut für Computing NIC, 15738 Zeuthen, Germany

${ }^{e}$ Deutsches Elektronen-Synchrotron DESY, 22603 Hamburg, Germany

${ }^{f}$ Dep. of Physics and Astronomy, University of Kentucky, Lexington, KY 40506-0055, USA

${ }^{g}$ Institut für theoretische Physik, Freie Universität Berlin, 14196 Berlin, Germany

${ }^{h}$ Institut für theoretische Physik, Universität Tübingen, 72076 Tübingen, Germany

E-mail: ilgenfri@physik.hu-berlin.de

\begin{abstract}
We present selected recent results of the QCDSF collaboration on the localization and dimensionality of low overlap eigenmodes and of the topological density in the quenched $S U(3)$ vacuum. We discuss the correlations between the topological structure revealed by overlap fermions without filtering and the confining monopole and P-vortex structure obtained in the Indirect Maximal Center Gauge.
\end{abstract}

The XXV International Symposium on Lattice Field Theory July 30 - August 4, 2007

Regensburg, Germany

\footnotetext{
* Speaker.
} 


\section{Motivation}

Overlap fermions [1] possess exact chiral symmetry on the lattice, realize the Atiyah-Singer index theorem [2] and provide a local definition of the topological charge density [3]. This makes them an ideal tool for investigating the chiral and topological QCD vacuum structure.

In this talk we shall summarize some results of a recent extended study [4] of the vacuum structure of quenched QCD at zero temperature. One of the lessons is that there exists a whole family of topological descriptions, ranging from an UV filtered density (characterized by a cut-off scale $\lambda_{\text {cut }}$ and sign-coherent selfdual clusters) to a topological density with high $O(a)$ resolution (also called "all-scale density") forming global, sign-coherent lower-dimensional structures [5]. The other lesson is that, apart from the overall chirality that clearly distinguishes zero and nonzero modes, the localization features are smoothly changing from one to the others. The non-zero modes, through the local chirality, still feel the (filtered) topological background.

This talk focuses on two aspects of Ref. [4], the localization of the low-lying eigenmodes and of the all-scale topological density. The localization properties of the low-lying modes $[6,7,8,9]$ have attracted interest since they are hypothetically pinned down on singular defects [10] which are responsible for confinement. Candidates for this role are monopoles and vortices as located by Abelian or center projection (for a review see [11]). The localization of the all-scale topological density has also been considered [6] for similar reasons. In particular, peaks are expected on vortex intersections etc. and usually searched for by zero modes $[12,13]$. We stress that the mechanism behind the formation of the peculiar singular and global structure [5] appearing at low density is unknown. This structure is necessary for the negativity [14, 8, 4] of the two-point function $C(x-y)=\langle q(x) q(y)\rangle$ required by reflection positivity [15]. This aspect of topological charge is complementary to the instanton-like clustering of the UV filtered density in approximately (anti-)selfdual domains [4]. Thus, the low-lying modes and the (unfiltered) topological density can be seen in closer relation to the mechanism of confinement. On a macroscopical level, such a relation is well established: the removal of vortices or monopoles from lattice configurations simultaneously destroys the topological charge and restores chiral symmetry $[16,17,18]$.

\section{Localization of overlap eigenmodes}

We use the massless Neuberger [1] overlap Dirac operator

$$
D_{o v}(0)=\frac{\rho}{a}\left(1+D_{W} / \sqrt{D_{W}^{\dagger} D_{W}}\right), \quad \text { with } D_{W}=M-\frac{\rho}{a},
$$

the Wilson Dirac operator with hopping term $M$ and negative mass $\rho / a$. The quenched ensembles of [4] were generated by the Lüscher-Weisz action, for $\beta=8.45$ on lattices $12^{3} \times 24,16^{3} \times 32$ and $24^{3} \times 48$, for $\beta=8.1$ on $12^{3} \times 24$ and for $\beta=8.0$ on $16^{3} \times 32$. First results have been reported by Y. Koma [8] at Lattice 2005. At that time, we estimated the dimension of zero modes and non-zero modes in the lowest bins of the spectrum from the volume $V$ dependence of the Inverse Participation Ratios (IPR), IPR $=V I_{2}=V \sum_{x}\left|\psi_{\lambda}(x)\right|^{4}$, averaged over the respective modes. The average IPR should follow a power law

$$
\langle I P R\rangle=c_{1}+c_{2} V^{1-d^{*} / 4},
$$




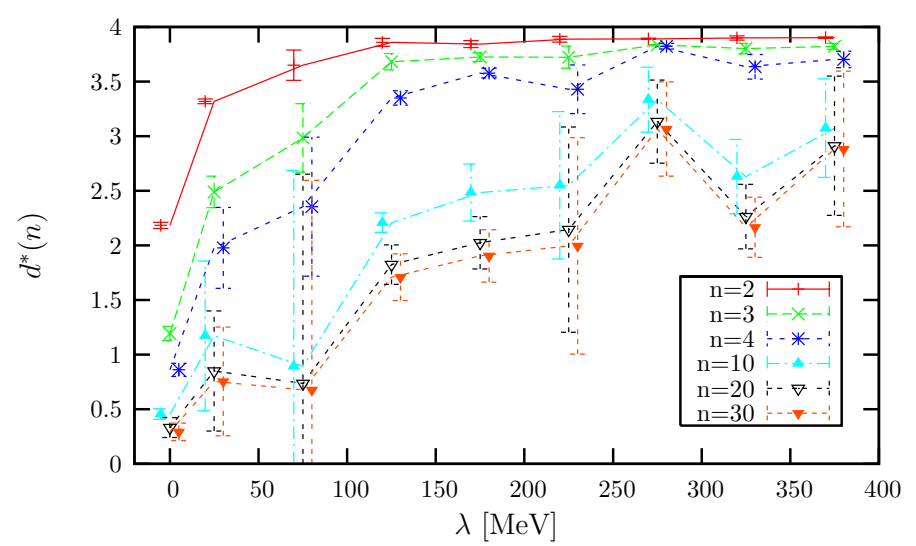

Figure 1: Fractal dimension $d^{*}(n)$ obtained from fits of the volume dependence of the averages of $I_{n}$, for zero modes and for non-zero modes in bins $\Delta \lambda=50 \mathrm{MeV}$, for three ensembles with the same $\beta=8.45$ and different volumes.

allowing to infer the fractal dimension $d^{*}$. We concluded [8] that zero modes are $d^{*}=2$ and next-to-zero modes $d^{*}=3$ dimensional. Now we refine this statement by considering generalized IPR's [19]. With their help one should be able to find lower dimensions for regions of higher density if a multifractal structure is physically realized. The second moment $I_{2}$ of the scalar density $p(x)=\left|\psi_{\lambda}(x)\right|^{2}$ is replaced by higher one, $I_{n}=\sum_{x}\left|\psi_{\lambda}(x)\right|^{2 n}$, such that a sequence of dimensions $d^{*}(n)$ can be extracted from the volume scaling of $\left\langle I_{n}\right\rangle \propto L^{d^{*}(n)(n-1)}$. The result of this analysis is shown in Fig. 1. This plot shows that the regions of higher scalar density are lower dimensional (between $d^{*}=0$ and 1). There is a gradual change of the localization properties from zero modes to non-zero modes.

In Ref. [4] we have described methods to estimate the dimension of an arbitrary distribution at any level of the density. Both methods assume a cluster analysis already made to separate peaks of the distribution from the rest of the system. The emerging set of connected clusters, as function of a running parameter, e.g. the lower density cut-off for the clusters, characterizes the distribution [4]. In the random walker method, for random walkers moving inside a cluster, the return probability to the cluster center, $P(0, t) \propto t^{-d^{*} / 2}$, provides an estimate of the dimension $d^{*}$ depending on the adopted cut-off. Another cut-off dependent dimension $d^{*}$ can be inferred, in the covering-sphere method, from the growth (from 0 to 1 ) of the cumulative fraction $Q_{\text {cumulative }}$ of a cluster's total charge that is covered by a 4D sphere of radius $R$. This growth begins $Q_{\text {cumulative }} \propto R^{d^{*}}[4]^{1}$.

Fig. 2 shows for selected modes in an ensemble of 170 lattices $16^{3} \times 32$ at $\beta=8.45$ the number of clusters (i.e. separate maxima) on the left and the effective dimension $d^{*}$ of the clusters on the right as function of the cut-off $p_{\text {cut }}$. The dimension was estimated by the random walker method. Percolation, that is not shown here, sets in between $p_{\text {cut }} / p_{\max }<0.1$, i.e. rather low for the zero modes, and $p_{\text {cut }} / p_{\max }=0.3$ for the 120 -th modes.

We conclude that all modes, including the zero modes, percolate at sufficiently low density. If cut at an average level of density, 5 to 20 different peaks are discernible, depending on the mode. The zero modes, before finally percolating, too, do not exceed a dimension $d^{*}=2$ as long as $p_{\text {cut }} / p_{\max }>0.2$.

\footnotetext{
${ }^{1}$ Although the results are similar, the effective dimensions obtained by the two methods do not strictly agree.
} 

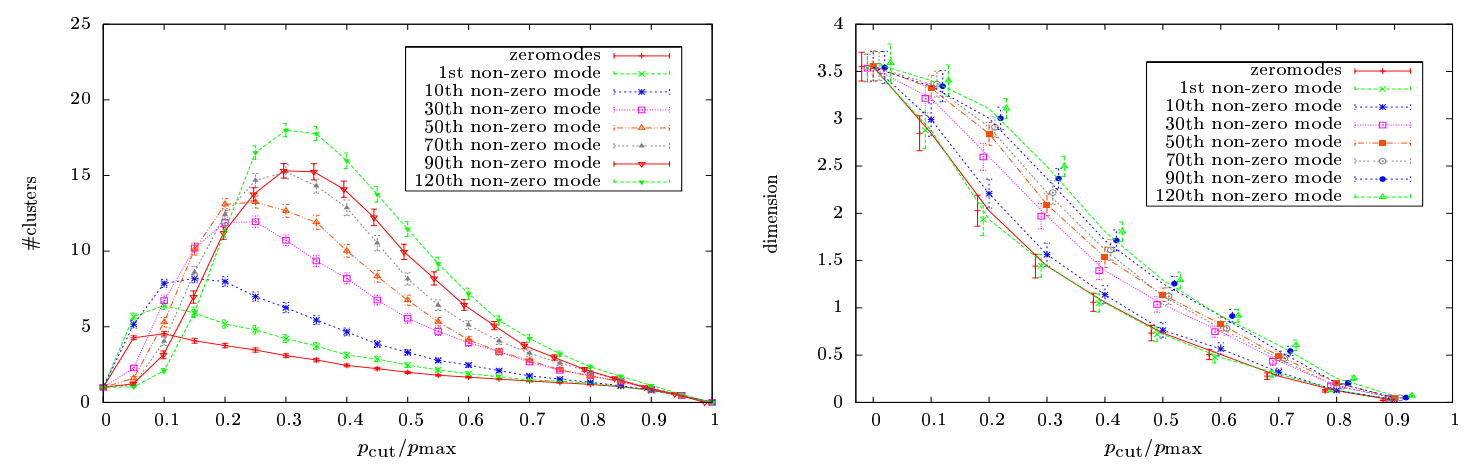

Figure 2: Cluster analysis of selected eigenmodes, averaged over the ensemble mentioned in the text, as function of the cut-off $p_{\text {cut }} / p_{\max }$ for the scalar density. Left: number of clusters in the mode; right: effective dimension $d^{*}$ of the mode.

\section{Localization of topological charge}

For a Dirac operator satisfying the Ginsparg-Wilson relation, the topological charge density can be expressed as a local trace (with the massless overlap operator $D(0)$ )

$$
q(x)=-\operatorname{tr}\left[\gamma_{5}\left(1-\frac{a}{2} D(0 ; x, x)\right)\right], \quad Q=\sum_{x} q(x)
$$

over color and spinor indices. The UV filtered densities are obtained by casting this into a spectral sum and using a mode-truncation $|\lambda|<\lambda_{\text {cut }}$. Without truncation, we have evaluated the density only for a small subset of two of our ensembles (53 configurations $12^{3} \times 24$ for $\beta=8.1$ and 5 configurations $16^{3} \times 32$ for $\beta=8.45$ ) representing almost equal volume. Fig. 3 shows, analogously to the last figure, the cluster composition and dimension of the unfiltered topological density as function of $q_{\text {cut }}$ (meant as a cut-off for $|q(x)|$ ) for the two lattices. The left plot shows that for $q_{\text {cut }} / q_{\max }>0.5$ only few isolated spikes are detected. They do not have a sufficient extension that a power-law decay of $P(0, t)$ could be determined ("0-dimensional"). The right plot shows that for $q_{\text {cut }} / q_{\max }<0.5$ the dimension starts growing to $d^{*}=2.5$. At the maxima ${ }^{2}$ of multiplicity, at
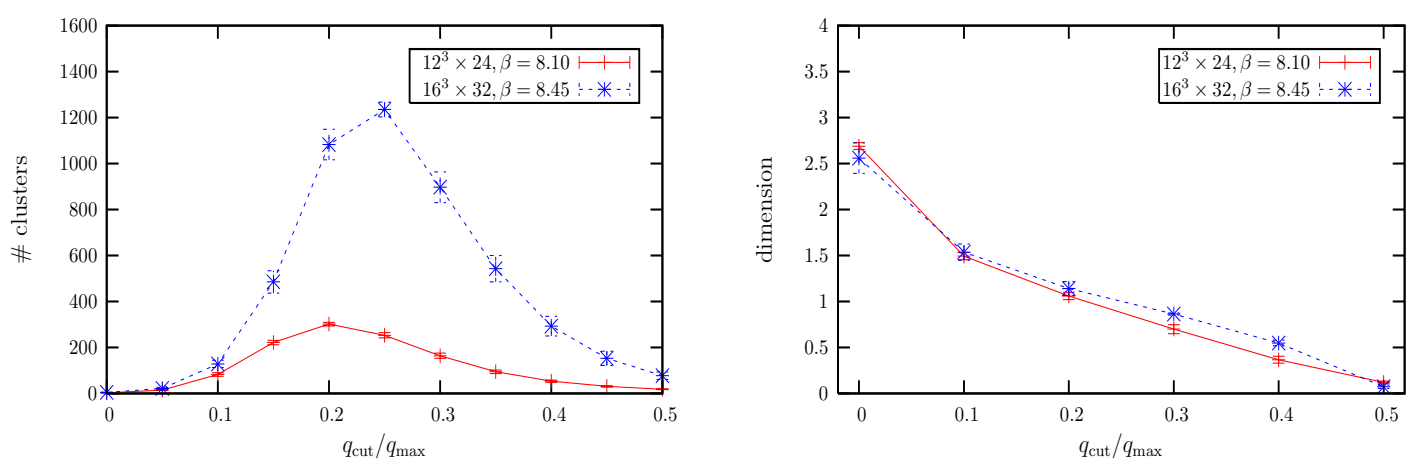

Figure 3: Cluster analysis of the all-scale topological density for the $16^{3} \times 32$ lattice at $\beta=8.45$ and the $12^{3} \times 24$ lattice at $\beta=8.1$ as function of the cut-off $q_{\text {cut }} / q_{\max }$. Left: number of clusters; right: effective dimension of the clusters from the random walker method.

\footnotetext{
${ }^{2}$ With $a \rightarrow 0$, the maximal number of clusters rises strongly.
} 

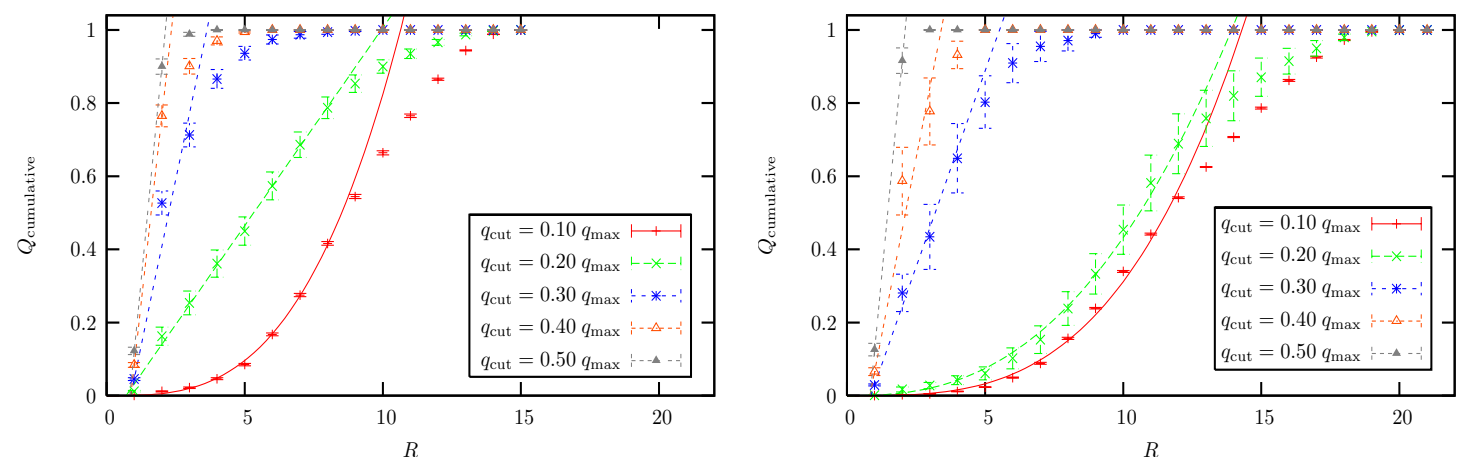

Figure 4: The ratio $Q_{\text {cumulative }}(R)$ of the charge covered by the sphere to the full cluster charge for the largest cluster is shown as function of $R$ for various cut-off values, for the $12^{3} \times 24$ lattice at $\beta=8.1$ (left) and the $16^{3} \times 32$ lattice at $\beta=8.45$ (right). Note the abrupt change at $q_{\mathrm{cut}} / q_{\max }=0.2$ (left) and 0.25 (right).

$q_{\text {cut }} / q_{\max }=0.2$ (for the coarse) and 0.25 (for the fine lattice), we find the same $d^{*}=1$. Close to these maxima the covering sphere method also finds a change shown in Fig. 4, signaling the onset of percolation. Below $q_{\text {cut }} / q_{\max }<0.05$ the cluster composition goes over to two sign-coherent global clusters of charge $Q_{+}$and $Q_{-}$which fill the volume and build the total charge $Q$ of the configuration. A distance between clusters, $C, C^{\prime}$, can be defined as $\Delta_{C, C^{\prime}}=\max _{x \in C}\left(\min _{y \in C^{\prime}}|x-y|\right)$. When the percolation is complete, the two remaining clusters have $\Delta \approx 2 a$, i.e. are closely intertwining each other.

\section{Lower dimensional objects and confining vacuum defects}

Here we shall offer an explanation for the even lower-dimensional local clusters of the unfiltered topological charge inside the global clusters (with $d^{*} \approx 2.5$ ). For $1<d^{*}<2$, monopoles and vortices are good candidates to cause the localization of charge. These are the two types of confining defects which are intimately connected [17]. If one sort is removed, the other one disappears together with the topological charge (all zero modes) and the non-zero modes close to $\lambda=0$ [18]. To demonstrate the correlation we have used the Indirect Maximal Center Gauge (IMCG) [20]. In a first step the Maximally Abelian Gauge (MAG) is accomplished, followed by Abelian projection: each MAG-fixed ${ }^{g} U_{\text {link }} \in S U(3)$ is replaced by the closest diagonal matrix $D_{\text {link }} \in S U(3)$. The norm $\left\|{ }^{g} U_{\text {link }}-D_{\text {link }}\right\|$ is called non-Abelianicity. The monopole worldlines are located on cubes (i.e. links of the dual lattice) where the Abelian magnetic charges $\left(m_{c}^{(1)}, m_{c}^{(2)}, m_{c}^{3}\right)\left(\sum_{k} m_{c}^{(k)}=0\right)$ are not all vanishing. In a second step, within residual Abelian gauge transformations $h \in U(1)^{2}$, we find the Maximal Center Gauge (MCG) which brings ${ }^{h} D_{\text {link }}$ as close as possible to multiples of unity, $z_{\text {link }} \times \operatorname{diag}(1,1,1)$ with $z_{\text {link }} \in Z(3)$ being the links after center projection. Center plaquettes, for which $p=\Pi_{\text {link } \in \partial p} z_{\text {link }} \neq 1$, mark the presence of a vortex that is geometrically located on the dual ("vortex") plaquette * $p$. A peculiarity of $S U(3)$ compared to $S U(2)$ is vortex splitting.

Density and connectivity describe the "vortex matter" corresponding to some given gauge field ensemble. For $\beta=8.45$ we find the probability for a dual site to be adjacent to $n$ vortex plaquettes as shown by the histogram in the left of Fig. 5 . Here $87 \%$ of sites belong to the bulk $(n=0), 4.3 \%$ are adjacent to 3 plaquettes (corner), $3.8 \%$ to 4 plaquettes (planar vortex) etc. The probability for a dual link to be adjacent to $n$ vortex plaquettes is shown in the histogram on the right of Fig. 5. This 

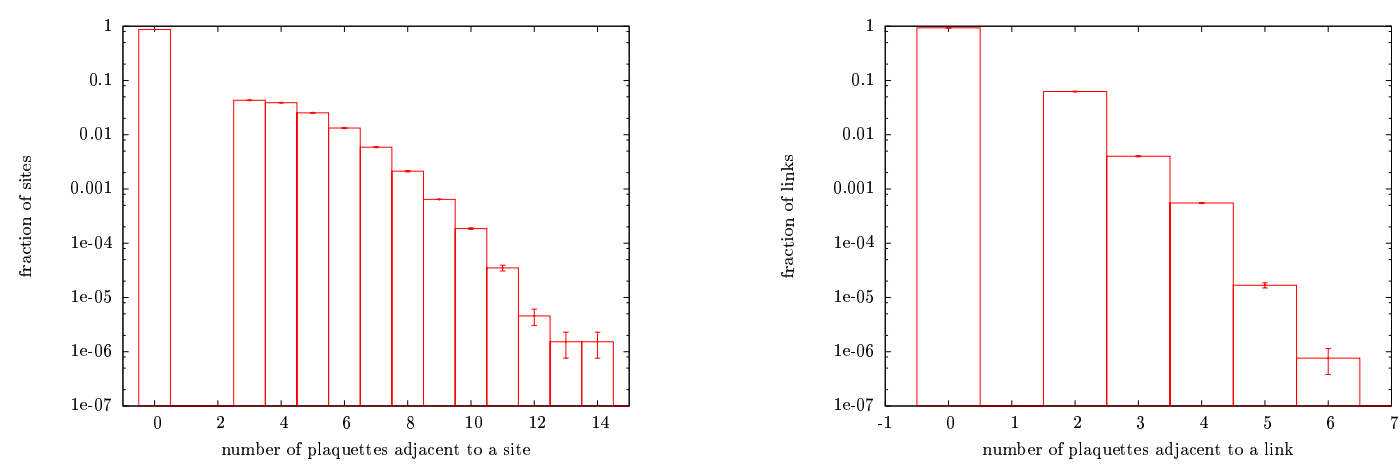

Figure 5: The connectivity of the vortex structure. Left: histogram of dual sites w.r.t. the number $n$ of adjacent vortex plaquettes; Right: histogram for dual links w.r.t. the number $n$ of adjacent vortex plaquettes.

is an important input for the construction of a realistic effective vortex model [21] for confinement. Thus $93 \%$ of the links belong to the bulk $(n=0), 6.25 \%$ are adjacent to $n=2$ plaquettes, $0.4 \%$ to $n=3$ plaquettes (branching) etc.

Close to the monopoles, the non-Abelianicity and the modulus of the topological density $|q(x)|$ show an excess above their bulk averages. Thus they are positively correlated. Fig. 6 illustrates the enhanced probability to find a site of the original lattice close to a monopole and/or vortex if for the (unfiltered) topological density at the site $|q(x)|>0.2 q_{\max }$ is fulfilled. This proves that the confining defects are the preferred location for topological charge.

\section{Summary}

We have summarized the localization of eigenmodes and of the unfiltered all-scale topological density $q(x)$ provided by our recent investigation of the vacuum structure based on the overlap operator [4]. In addition, we have presented first results relating the low dimensionality of $q(x)$ at densities above the percolation threshold to a local correlation with monopoles and vortices detected in the course of IMCG. More results and corresponding observations concerning the lowest modes will be published elsewhere [22].
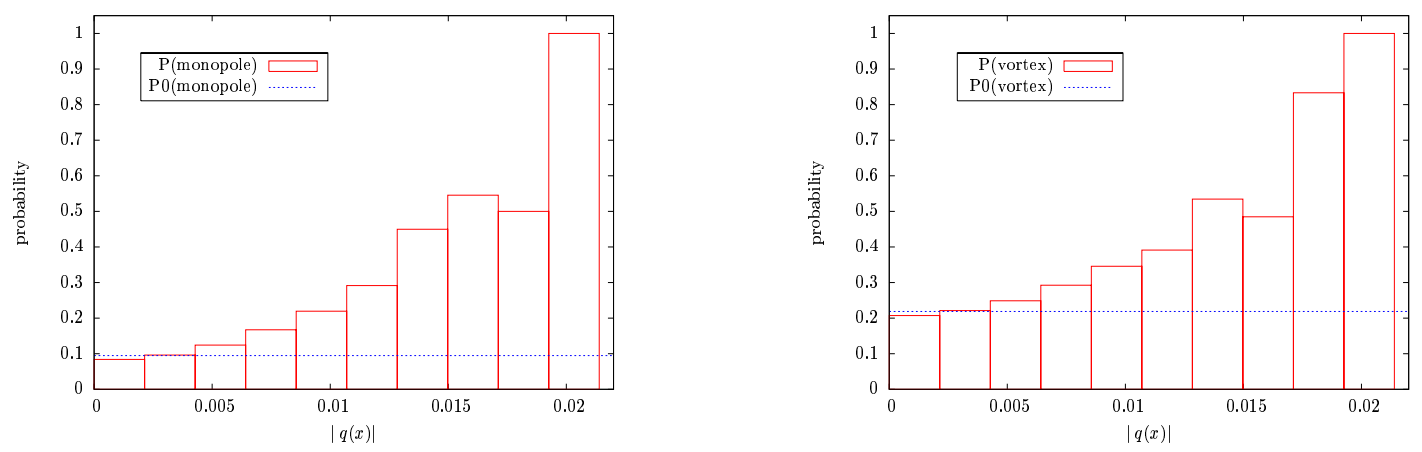

Figure 6: The probability $\mathrm{P}$ for a site of the original lattice to be adjacent (closest) to a monopole (left) and to a vortex (right) depending on the unfiltered topological density. The horizontal blue lines show the $a$ priori probability $\mathrm{P} 0$ for a site to be close to a monopole or vortex. The histogram covers the interval from 0 to $q_{\max }$. 


\section{Acknowledgements}

The numerical overlap calculations have been performed on the IBM p690 at HLRN (Berlin) and NIC (Jülich), as well as on the PC farms at DESY Zeuthen and LRZ Munich. We thank these institutions for support. Part of this work is supported by DFG under contract FOR 465 (Forschergruppe Gitter-Hadronen Phänomenologie).

\section{References}

[1] H. Neuberger, Phys. Lett. B417, 141 (1998), [hep-lat/ 9707022 ]; Phys. Lett. B427, 353 (1998), [hep-lat/9801031].

[2] P. Hasenfratz, V. Laliena and F. Niedermayer, Phys. Lett. B427, 125 (1998), [hep-lat/9801021].

[3] F. Niedermayer, Nucl. Phys. Proc. Suppl. 73, 105 (1999), [hep-lat/9810026].

[4] E.-M. Ilgenfritz et al., Phys. Rev. D76, 034506 (2007), 0705.0018 [hep-lat ] .

[5] I. Horvath et al., Phys. Rev. D68, 114505 (2003), [hep-lat/ 030200 9]; Phys. Lett. B612, 21 (2005), [hep-lat/0501025].

[6] C. Aubin et al., [MILC collaboration], Nucl. Phys. Proc. Suppl. 140, 626 (2005), [hep-lat/0410024].

[7] F.V. Gubarev, S.M. Morozov, M.I. Polikarpov and V.I. Zakharov, JETP Lett. 82, 343 (2005), [hep-lat/0505016].

[8] Y. Koma et al., PoS (LAT2005) 300 (2006), [hep-lat/ 0509164$].$

[9] Ph. de Forcrand, AIP Conf. Proc. 892, 29 (2007), [hep-lat/ 0611034 ].

[10] V.I. Zakharov, Phys. Atom. Nucl. 68, 573 (2005), [hep-lat/ 041003 4]; Braz. J. Phys. 37, 165 (2007), [hep-ph/0612342].

[11] J. Greensite, Prog. Part. Nucl. Phys. 51, 1 (2003), [hep-lat/0301023].

[12] J. Gattnar et al., Nucl. Phys. B716, 105 (2005), [hep-lat / 0412032$].$

[13] S. Solbrig et al., POS (LAT2005) 301 (2006), [hep-lat/0509052].

[14] I. Horvath et al., Phys. Lett. B617, 49 (2005), [hep-lat/ 0504005 ].

[15] E. Seiler, Phys. Lett. B525, 355 (2002), [hep-th/0111125]; M. Aguado and E. Seiler, Phys. Rev. D72, 094502 (2005), [hep-lat/ 0503015$].$

[16] Ph. de Forcrand and M. D'Elia, Phys. Rev. Lett. 82, 4582 (1999), [hep-lat/9901020].

[17] P.Yu. Boyko et al., Nucl. Phys. B756, 71 (2006), [hep-lat/ 0607003$].$

[18] V.G. Bornyakov et al., 0708.3335 [hep-lat].

[19] V.E. Kravtsov, [hep-lat/9603166].

[20] L. Del Debbio et al., Phys. Rev. D58, 094501 (1998), [hep-lat/9801027]; J. Greensite, Eur. Phys. J. Special Topics 140, 1 (2007).

[21] M. Quandt, H. Reinhardt and M. Engelhardt, Phys. Rev. D71, 054026 (2005), [hep-lat/0412033].

[22] E.-M. Ilgenfritz et al., in preparation. 